\title{
Avian diversity and their status in and around Bhindawas bird sanctuary, Haryana (India)
}

\author{
Girish Chopra, Deepak Rai and Jyoti* \\ Department of Zoology, Kurukshetra University, Kurukshetra-136119 (Haryana), INDIA \\ *Corresponding author. E-mail: jyotiikukz@gmail.com \\ Received: August 14, 2016; Revised received: December 12, 2016; Accepted: July 25, 2017
}

\begin{abstract}
One year survey conducted in and around Bhindawas bird sanctuary in district Jhajjar, Haryana (India) from January, 2015 to December, 2015; revealed a total of 104 bird species belonging to 15 orders and 39 families. Of these, 67 bird species were resident, 32 species were winter migrants and only 5 species were summer migrants. Based on their frequency of sighting, 33 bird species were categorized as Abundant, 27 species as Common, 32 species as Uncommon and 12 species as Rare. Maximum number of species belonged to order Passeriformes and least number of species belonged to order Podicipediformes and Strigiformes. Among the recorded birds, 4 species namely, Darter, Anhinga melanogaster; Black-necked Stork, Ephippiorhynchus asiaticus; Oriental White Ibis, Threskiornis melanocephalus; and Alexandrine Parakeet, Psittacula eupatria were near threatened and 2 species namely, Common, Pochard, Aythya ferina and Sarus Crane, Grus antigone were vulnerable. In early 1990s, a study was conducted on Bhindawas bird sanctuary and thereafter, a great deal of infrastructure, development and urbanization has occurred. However, scanty information is available on the avian diversity periodic monitoring of Bhindawas bird sanctuary, Haryana. Therefore, present study was planned to monitor and document the avian species.
\end{abstract}

Keywords: Abundant, Bhindawas bird sanctuary, Diversity, Migrants, Resident

\section{INTRODUCTION}

Birds are the most beautiful creatures on the planet; they exhibit a great variety of specific features enabling them to occupy numerous habitats, i.e. shrubby, mixed forest and aquatic habitat. Besides, birds play roles as pollinators, scavengers, predators, bioindicator of ecosystem and they also function as early warning system (Dhadse et al., 2009). From time to time, various ornithologists have conducted studies on birds in different regions as well as in various protected areas of India (Barua and Sharma, 1999; Mahabal, 2000; Aravind et al., 2001; Urfi, 2003; Kumar, 2007; Harisha and Hosetti, 2009; Barsal and Inskipp, 2009; Maity et al., 2010; Saikia and Saikia, 2011; Lameed, 2012; Bibi and Ali, 2013; Wijesundara and Wijesundara, 2014; Abbas et al., 2014; Indika and Mahaulpatha, 2015).

In Haryana, Bhindawas Bird Sanctuary is the largest wetland having Eucalyptus, Acacia, Azadirachta and Zizyphus which are the main trees planted around the lake. Cormorants, egrets, storks etc. use them for roosting and nesting. In the last few years, huge anthropogenic pressure is being witnessed in this region. Keeping in view the conservational values of sanctuary, the present study was conducted to prepare the checklist of birds and also to list various factors which may be responsible for the affecting avian diversity in the Bhindawas bird sanctuary.

\section{MATERIALS AND METHODS}

Study area: Bhindawas bird sanctuary is located 25 kms south-east of Jhajjar $\left(28^{\circ} 37^{\prime} \mathrm{N}\right.$ and $\left.76^{\circ} 40^{\prime} \mathrm{E}\right)$ and about $80 \mathrm{kms}$ north-west of Delhi. This Sanctuary spreads over an area of 1016.94 acres. It was notified as wildlife Sanctuary on $7^{\text {th }}$ May, 1986. The area also has a lake with a periphery of about $12 \mathrm{kms}$. The Sanctuary premises are surrounded by seven villages, namely, Kanwah, Bilochpura, Nawada, Redhuwas, Shanjabanpur, Kunjah and Chadwana. The climate of the Sanctuary is dry with an intensely hot summer and a cold winter (Fig. 1, 2).

Methodology: To record the avian diversity, periodic fortnightly visits were conducted from January 2015 to December 2015. During each visit, data was recorded in the morning by using line transect method (Gaston, 1975; Sale and Berkmuller, 1988). Birds were observed using the binoculars (Nikon Monarch 8x42) and photographs were taken by digital camera (Canon 1200D). The Photographs were subsequently identified using field guide by Grimmett et al. (1999). Classification of the observed bird species was done following Manakandan and Pittie (2001). Abundance status of the bird, on the basis of the frequency of sighting, i.e., Abundant (90-100\%), Common (60-90\%), Uncommon (20-60\%), Rare (less than 20\%) was observed following the terminology of Srinivasulu and Nagulu (2001). Residential / migratory status of the birds was assessed 

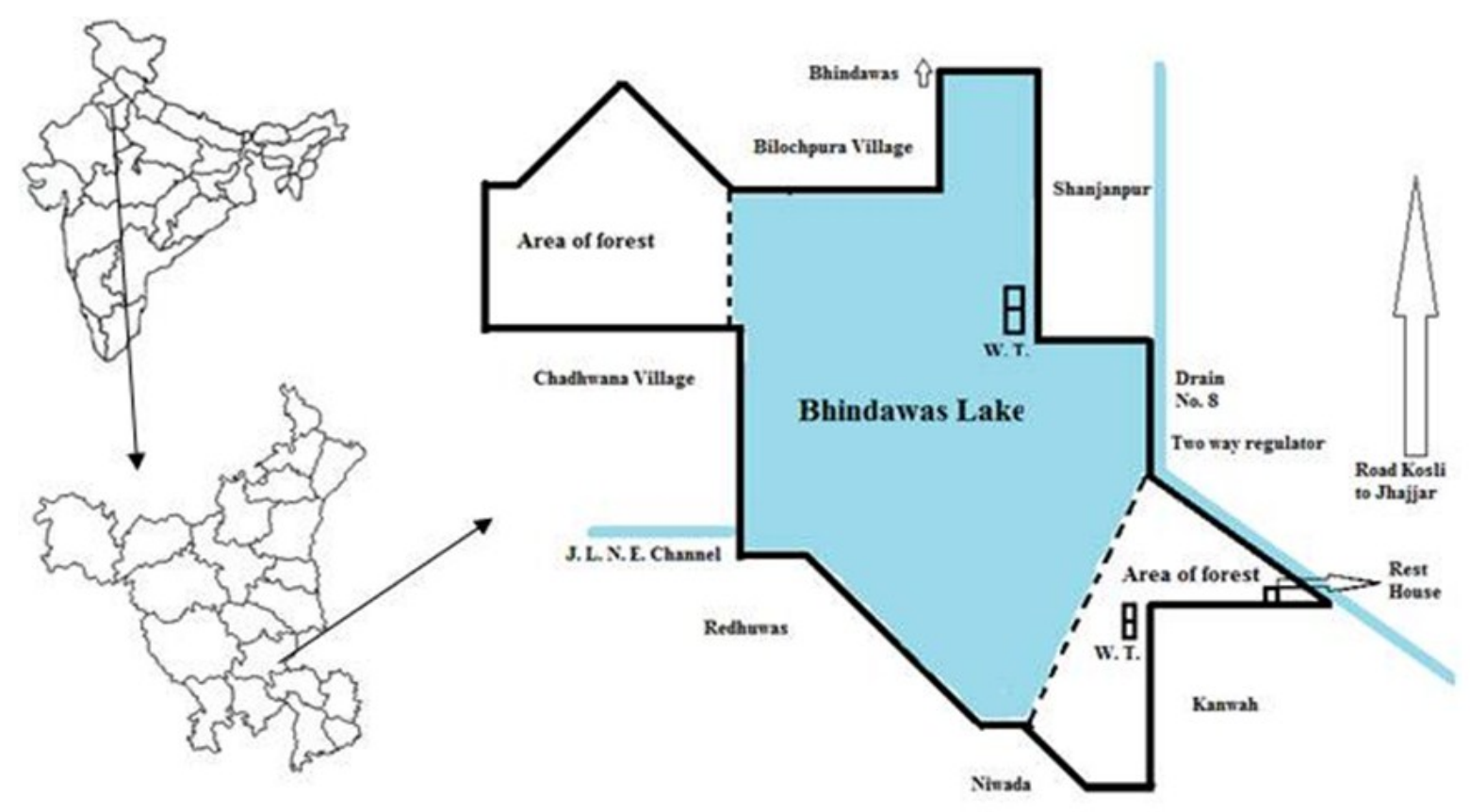

Fig. 1. Location and Diagrammatic presentation of Bhindawas bird sanctuary in district Jhajjar, Haryana.

strictly with reference to the study area on the basis of presence or absence method following Ali and Ripley, (1987). Later bird species were categorized as 'Resident'(R) and 'Migratory'- 'Local migrants' (LM), 'Summer migrants' (SM) and 'Winter migrants' (WM).

\section{RESULTS AND DISCUSSION}

In the present study, a total 104 species of birds were identified in and around Bhindawas bird sanctuary during January 2015 to December 2015 (Table 1). Recorded bird species belonged to 15 orders, namely, Podicipediformes, Pelecaniformes, Ciconiiformes, Anseriformes, Falconiformes, Galliformes, Gruiiformes, Charadriiformes, Columbiformes, Psittaciformes, Cuculiformes, Strigiformes, Coraciiformes, Piciformes and Passeriformes. The study revealed that maximum number of species belonged to order Passeriformes (31.73\%) followed by Anseriformes (12.5\%), Charadriiformes (11.54\%), Ciconiiformes (10.58\%), Coraciiformes (6.73\%), Gruiiformes and Columbiformes (4.81\% each), Pelecaniformes, Falconiformes $(3.85 \%$ each), Galliformes, Psittaciformes, $\mathrm{Cu}$ cliformes and Piciformes (1.92\% each) and Podicipediformes and Strigiformes with least number of species with $0.96 \%$ each (Fig. 3). Earlier studies of Pawar (2011), in mangroves of Uran (Raigad), Navi Mumbai, Maharashtra, West coast of India and Chilke (2012), in and around Bamanwada lake of Rajura, district- Chandrapur (Maharashtra) have also reported that Passeriformes was the most dominant order representing maximum number of species. In their studies, most of the avian species were resident $(\mathrm{R})$ and rest were migrant species. Beresford et al. (2005) explained the reason of high diversity of Passerine birds to their ability to use various kinds of habitats and to have large variety of food items viz. grains, seeds, floral buds, fruits, nectar and invertebrates. Order and family wise distribution of avian species has also been presented in Table 2.

Study revealed heterogeneous distribution of birds in different types of habitats, viz., along roadside and the periphery of the lakes, inside the wetland, in and around agricultural fields, and in fallow lands. A total of 56 species were recorded on vegetation along roadside, electrical wires and the periphery of the lake, 51 species from wetland, 26 species form agricultural fields and 19 species from fallow lands (Fig. 4); of the total recorded avian fauna, 28 species were recorded from more than one type of habitats, 7 species were sighted from three type of habitats; of these, 3 species were sighted on roadside plantation, agricultural fields and fallow land; 3 species were noticed in wetland, agricultural fields and fallow land; one species was sighted on wetland, roadside plantation and fallow land, and 2 species namely, Cattle Egret and Indian Pond Heron were observed from all type of habitat. Earlier also Chopra and Sharma (2014) recorded 73 $(46.79 \%)$ species from roadside plantation, 36 $(23.08 \%)$ species from wetland, $24(15.38 \%)$ species from agricultural field and 23(14.74\%) species from orchards in lower Shivalik foothills.

Out of total, 67 species were resident, 32 species were winter migrants and 5 species were summer migrants 


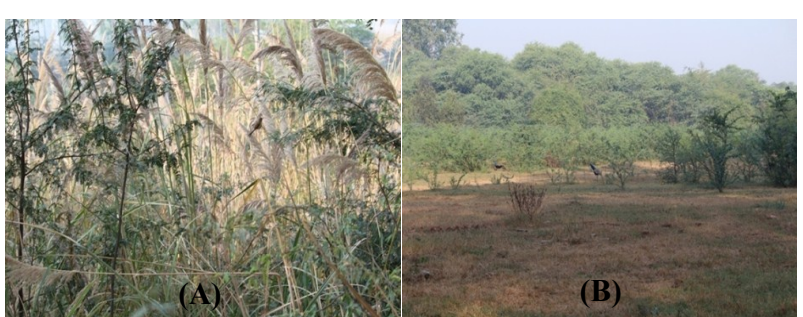

Fig. 2. (A) Vegetation of Bhindawas bird sanctuary and (B) Fallow land in the Bhindawas bird sanctuary.

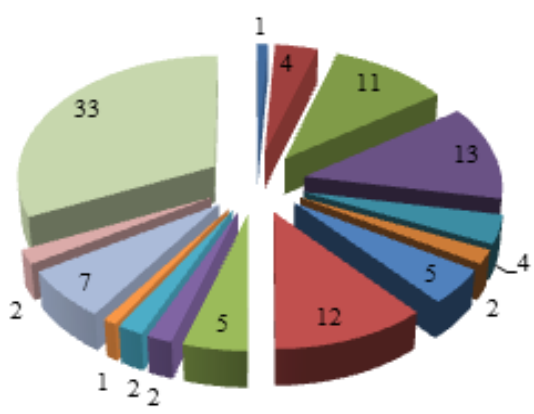

- Podicipediformes - Pelecaniformes - Ciconiiformes

- Anseriformes - Falconiformes - Galliformes - Gruilformes - Charadriiformes n Columbiformes - Psittaciformes - Cuculiformes = Strigiformes - Coraciiformes - Piciformes

= Passeriformes

Fig. 3. Pie chart representing number of avian species in different order in Bhindawas bird sanctuary.

(Fig. 4). In previous studies, maximum number of species were recorded in winter season due to more food availability and favorable climatic conditions for nesting and roosting at Jaikwadi reservoir, Paithan (Ghorade et al., 2014); minimum number of species were recorded during monsoon season and maximum during the winter season in Chandubi tectonic lake, Assam (Deka and Nath, 2013). Based on the frequency of their sighting, 33 species were abundant, 27 species were common, 32 species were uncommon and 12 species were rare (Fig. 4). Glossy Ibis, Plegadis falcinellus; Brahminy Shelduck, Tadorna ferruginea;
Mallard, Anas platyrhynchos; Black-shouldered Kite, Elanus caeruleus; Pied Harrier, Circus macrourus; Sarus Crane, Grus antigone; White-tailed Lapwing, Vanellus leucurus; Temminck's Stint, Calidris temminckii; Red Collared Dove, Streptopelia tranquebarica; Indian Grey Hornbill, Ocyceros birostris; Streakthroated Swallow, Hirundo fluvicola; Bluethroat, Luscinia svecica were 'rarely sighted' bird species in the study area.

Recently, IUCN Red list of endangered birds reveals in which 1375 bird species were recognized as globally threatened and India is ranked at the eighth position with 84 threatened bird species (Bird Life International, 2016). In the present study, 4 species were near threatened, namely, Darter Anhinga melanogaster, Black-necked Stork Ephippiorhynchus asiaticus, Oriental White Ibis Threskiornis melanocephalus and Alexandrine Parakeet Psittacula eupatria) and 2 species were vulnerable (Common Pochard Aythya ferina and Sarus Crane Grus antigone) and 98 species were least concerned (Fig. 4).

During the study period, lake was found now infested with Water Hyacinth Eichhornia crassipes and blue green algae, indicating the enriched nutrient loading in the lake. The possible reason may be nutrient loading from nearby agricultural field. Also, great deal of infrastructure development, increased urbanization, regular cutting of grass from the region by villagers and increasing pace of tourists being close to Delhi, the capital of India, are some of the factors which might affect avian diversity, in particular, in the region in near future.

\section{Conclusion}

Bhindawas bird sanctuary has rich avian fauna, beside this, it also supports some other mammalian species like Neelgai, Jungle Cat, Monkey and Rabbit. A total 104 species of birds were recorded, among the observed avian species some endangered species were

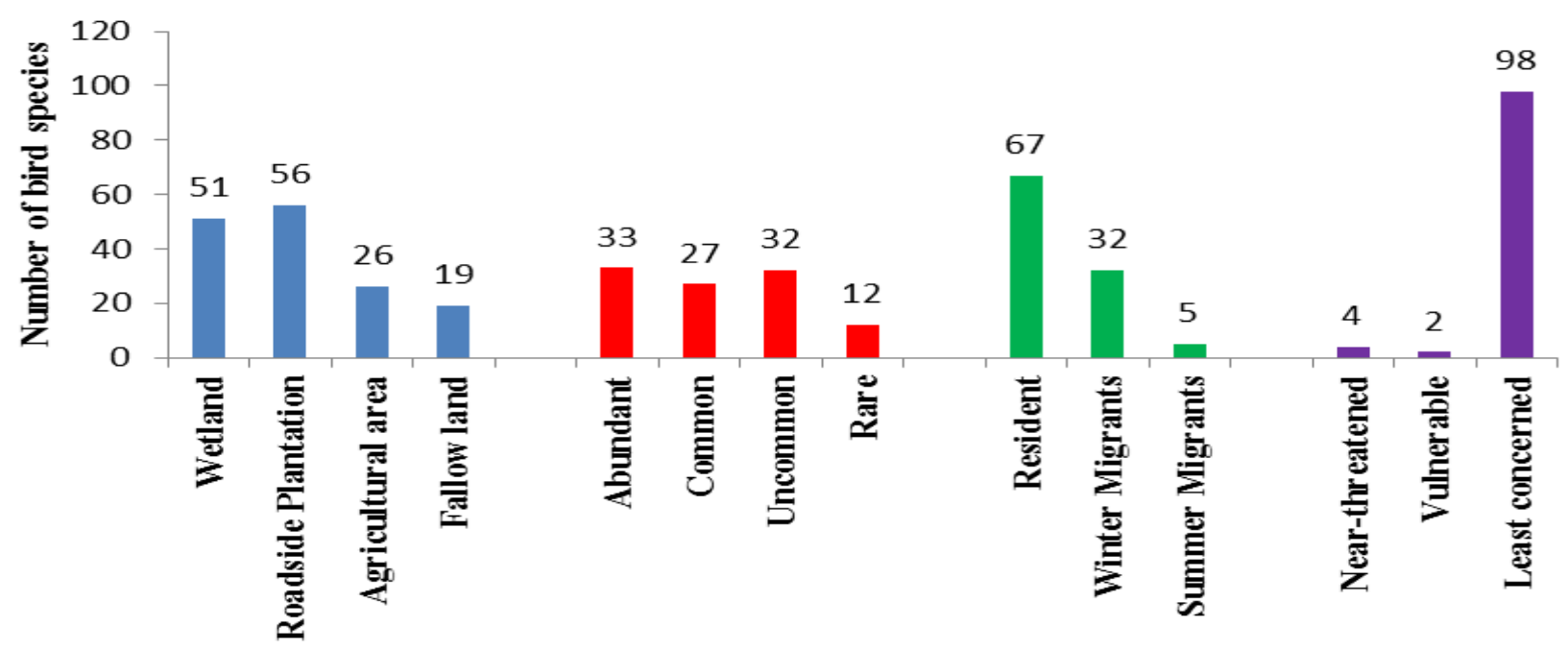

Fig. 4. Bar diagram representing the status of bird species from January 2015 to December 2015 in Bhindawas bird sanctuary. 
Girish Chopra et al. / J. Appl. \& Nat. Sci. 9 (3): 1475 - 1481 (2017)

Table 1. List of birds recorded from Bhindawas bird sanctuary during January 2015 to December 2015.

\begin{tabular}{|c|c|c|c|c|c|}
\hline $\begin{array}{lll}\begin{array}{l}\text { Family/S. No. of } \\
\text { species }\end{array} & \\
\end{array}$ & Common name & Scientific name & $\begin{array}{l}\text { Abundance } \\
\text { status }\end{array}$ & $\begin{array}{l}\text { Residen- } \\
\text { tial status }\end{array}$ & $\begin{array}{l}\text { IUCN } \\
\text { Status } \\
\end{array}$ \\
\hline \multicolumn{6}{|l|}{ Podicipedidae } \\
\hline 1 & Little Grebe & Tachybaptus ruficollis & $\mathrm{UC}$ & $\mathrm{R}$ & LC \\
\hline \multicolumn{6}{|l|}{ Phalacrocoracidae } \\
\hline 2 & Little Cormorant & Phalacrocorax niger & A & $\mathrm{R}$ & $\mathrm{LC}$ \\
\hline 3 & Indian Shag & Phalacrocorax fuscicollis & UC & $\mathrm{R}$ & $\mathrm{LC}$ \\
\hline 4 & Great Cormorant & Phalacrocorax carbo & UC & $\mathrm{R}$ & $\mathrm{LC}$ \\
\hline \multicolumn{6}{|l|}{ Anhingidae } \\
\hline 5 & Darter & Anhinga melanogaster & UC & $\mathrm{R}$ & NT \\
\hline \multicolumn{6}{|l|}{ Ardeidae } \\
\hline 6 & Little Egret & Egretta garzetta & $\mathrm{C}$ & $\mathrm{R}$ & $\mathrm{LC}$ \\
\hline 7 & Grey Heron & Ardea cinerea & $\mathrm{A}$ & $\mathrm{R}$ & $\mathrm{LC}$ \\
\hline 8 & Purple Heron & Ardea purpurea & $\mathrm{C}$ & $\mathrm{R}$ & $\mathrm{LC}$ \\
\hline 9 & Large Egret & Casmerodius albus & $\mathrm{UC}$ & $\mathrm{R}$ & $\mathrm{LC}$ \\
\hline 10 & Median Egret & Mesophoyx intermedia & $\mathrm{UC}$ & $\mathrm{R}$ & $\mathrm{LC}$ \\
\hline 11 & Cattle Egret & Bubulcus ibis & A & $\mathrm{R}$ & $\mathrm{LC}$ \\
\hline 12 & Indian Pond Heron & Ardeola grayii & $\mathrm{C}$ & $\mathrm{R}$ & $\mathrm{LC}$ \\
\hline \multicolumn{6}{|l|}{ Ciconiidae } \\
\hline 13 & Black-necked Stork & Ephippiorhynchus asiaticus & $\mathrm{C}$ & WM & NT \\
\hline \multicolumn{6}{|l|}{ Threskiornithidae } \\
\hline 14 & Glossy Ibis & Plegadis falcinellus & RA & WM & $\mathrm{LC}$ \\
\hline 15 & Oriental White Ibis & $\begin{array}{l}\text { Threskiornis melanocepha- } \\
\text { lus }\end{array}$ & $\mathrm{C}$ & WM & NT \\
\hline 16 & Black Ibis & Pseudibis papillosa & UC & $\mathrm{R}$ & $\mathrm{LC}$ \\
\hline \multicolumn{6}{|l|}{ Anatidae } \\
\hline 17 & Greylag Goose & Anser anser & $\mathrm{A}$ & WM & $\mathrm{LC}$ \\
\hline 18 & Bar-headed Goose & Anser indicus & A & WM & $\mathrm{LC}$ \\
\hline 19 & Brahminy Shelduck & Tadorna ferruginea & RA & WM & $\mathrm{LC}$ \\
\hline 20 & Comb Duck & Sarkidiornis melanotos & $\mathrm{UC}$ & WM & $\mathrm{LC}$ \\
\hline 21 & Cotton Teal & Nettapus coromandelianus & $\mathrm{UC}$ & SM & $\mathrm{LC}$ \\
\hline 22 & Gadwall & Anas strepera & A & WM & $\mathrm{LC}$ \\
\hline 23 & Eurasian Wigeon & Anas Penelope & A & WM & $\mathrm{LC}$ \\
\hline 24 & Mallard & Anas platyrhynchos & RA & WM & $\mathrm{LC}$ \\
\hline 25 & Spot-billed Duck & Anas poecilorhyncha & A & $\mathrm{R}$ & $\mathrm{LC}$ \\
\hline 26 & Northern Shoveller & Anas clypeata & A & WM & $\mathrm{LC}$ \\
\hline 27 & Northern Pintail & Anas acuta & $\mathrm{A}$ & WM & $\mathrm{LC}$ \\
\hline 28 & Garganey & Anas querquedula & $\mathrm{A}$ & WM & $\mathrm{LC}$ \\
\hline 29 & Common Pochard & Aythya ferina & $\mathrm{A}$ & WM & $\mathrm{V}$ \\
\hline \multicolumn{6}{|l|}{ Accipitridae } \\
\hline 30 & Black-shouldered Kite & Elanus caeruleus & RA & $\mathrm{R}$ & $\mathrm{LC}$ \\
\hline 31 & Black Kite & Milvus migrans & $\mathrm{C}$ & $\mathrm{R}$ & $\mathrm{LC}$ \\
\hline 32 & Crested Serpent-Eagle & Spilornis cheela & $\mathrm{UC}$ & $\mathrm{R}$ & $\mathrm{LC}$ \\
\hline 33 & Pied Harrier & Circus macrourus & RA & WM & $\mathrm{LC}$ \\
\hline \multicolumn{6}{|l|}{ Phasianidae } \\
\hline 34 & Grey Francolin & Francolinus pondicerianus & $\mathrm{C}$ & $\mathrm{R}$ & $\mathrm{LC}$ \\
\hline 35 & Indian Peafowl & Pavo cristatus & $\mathrm{A}$ & $\mathrm{R}$ & $\mathrm{LC}$ \\
\hline \multicolumn{6}{|l|}{ Gruidae } \\
\hline & Sarus Crane & Grus antigone & RA & $\mathrm{R}$ & $\mathrm{V}$ \\
\hline \multicolumn{6}{|l|}{ Rallidae } \\
\hline 37 & White-breasted Waterhen & Amaurornis phoenicurus & $\mathrm{C}$ & $\mathrm{R}$ & $\mathrm{LC}$ \\
\hline 38 & Purple Moorhen & Porphyrio porphyrio & $\mathrm{C}$ & $\mathrm{R}$ & $\mathrm{LC}$ \\
\hline 39 & Common Moorhen & Gallinula chloropus & $\mathrm{A}$ & WM & $\mathrm{LC}$ \\
\hline 40 & Common Coot & Fulica atra & $\mathrm{A}$ & WM & $\mathrm{LC}$ \\
\hline \multicolumn{6}{|l|}{ Jacanidae } \\
\hline 41 & Pheasant-tailed Jacana & Hydrophasianus chirurgus & $\mathrm{UC}$ & SM & LC \\
\hline 42 & Bronze-winged Jacana & Metopidius indicus & $\mathrm{C}$ & SM & $\mathrm{LC}$ \\
\hline \multicolumn{6}{|l|}{ Charadriidae } \\
\hline 43 & Little Ringed Plover & Charadrius dubius & $\mathrm{UC}$ & WM & $\mathrm{LC}$ \\
\hline 44 & Red-wattled Lapwing & Vanellus indicus & A & $\mathrm{R}$ & $\mathrm{LC}$ \\
\hline 45 & White-tailed Lapwing & Vanellus leucurus & RA & WM & $\mathrm{LC}$ \\
\hline
\end{tabular}

Contd.... 
Girish Chopra et al. / J. Appl. \& Nat. Sci. 9 (3): 1475 - 1481 (2017)

\begin{tabular}{|c|c|c|c|c|c|}
\hline \multicolumn{6}{|c|}{ Scolopacidae } \\
\hline 46 & Marsh Sandpiper & Tringa stagnatilis & A & WM & $\mathrm{LC}$ \\
\hline 47 & Spotted Redshank & Tringa erythropus & $\mathrm{C}$ & WM & $\mathrm{LC}$ \\
\hline 48 & Common Redshank & Tringa tetanus & $\mathrm{C}$ & WM & $\mathrm{LC}$ \\
\hline 49 & Little Stint & Calidris minuta & $\mathrm{UC}$ & WM & $\mathrm{LC}$ \\
\hline 50 & Temminck's Stint & Calidris temminckii & RA & WM & $\mathrm{LC}$ \\
\hline \multicolumn{6}{|c|}{ Recurvirostridae } \\
\hline 51 & Black-winged stilt & Himantopus himantopus & A & $\mathrm{R}$ & $\mathrm{LC}$ \\
\hline 52 & Pied Avocet & Recurvirostra avosetta & $\mathrm{UC}$ & WM & $\mathrm{LC}$ \\
\hline \multicolumn{6}{|c|}{ Columbidae } \\
\hline 53 & Blue Rock Pigeon & Columba livia & A & $\mathrm{R}$ & $\mathrm{LC}$ \\
\hline 54 & Spotted Dove & Streptopelia chinensis & $\mathrm{UC}$ & $\mathrm{R}$ & $\mathrm{LC}$ \\
\hline 55 & Eurassian Collared Dove & Streptopelia decaocto & $\mathrm{C}$ & $\mathrm{R}$ & $\mathrm{LC}$ \\
\hline 56 & Red Collared Dove & Streptopelia tranquebarica & RA & $\mathrm{R}$ & $\mathrm{LC}$ \\
\hline 57 & Laughing Dove & Stigmatopelia senegalensis & $\mathrm{A}$ & $\mathrm{R}$ & $\mathrm{LC}$ \\
\hline \multicolumn{6}{|c|}{ Psittacidae } \\
\hline 58 & Rose Ringed Parakeet & Psittacula krameri & A & $\mathrm{R}$ & $\mathrm{LC}$ \\
\hline 59 & Alexandrine Parakeet & Psittacula eupatria & $\mathrm{C}$ & $\mathrm{R}$ & NT \\
\hline \multicolumn{6}{|c|}{ Cuculidae } \\
\hline 60 & Asian Koel & Eudynamys scolopacea & $\mathrm{C}$ & SM & $\mathrm{LC}$ \\
\hline 61 & Greater Coucal & Centropus sinensis & A & $\mathrm{R}$ & $\mathrm{LC}$ \\
\hline \multicolumn{6}{|c|}{ Strigidae } \\
\hline 62 & Spotted Owlet & Athene brama & $\mathrm{C}$ & $\mathrm{R}$ & $\mathrm{LC}$ \\
\hline \multicolumn{6}{|c|}{ Alcedinidae } \\
\hline 63 & White breasted Kingfisher & Halcyon smyrnensis & A & $\mathrm{R}$ & $\mathrm{LC}$ \\
\hline 64 & Lesser Pied Kingfisher & Ceryle rudis & $\mathrm{UC}$ & WM & $\mathrm{LC}$ \\
\hline \multicolumn{6}{|c|}{ Meropidae } \\
\hline 65 & Small Bee-eater & Merops orientalis & $\mathrm{UC}$ & $\mathrm{R}$ & $\mathrm{LC}$ \\
\hline 66 & Blue-tailed Bee-eater & Merops philippinus & $\mathrm{UC}$ & SM & $\mathrm{LC}$ \\
\hline \multicolumn{6}{|c|}{ Coraciidae } \\
\hline 67 & Indian Roller & Coracias benghalensis & $\mathrm{UC}$ & $\mathrm{R}$ & $\mathrm{LC}$ \\
\hline \multicolumn{6}{|c|}{ Upupidae } \\
\hline 68 & Common Hoopoe & Upupa epops & $\mathrm{C}$ & $\mathrm{R}$ & $\mathrm{LC}$ \\
\hline \multicolumn{6}{|c|}{ Bucerotidae } \\
\hline 69 & Indian Grey Hornbill & Ocyceros birostris & RA & $\mathrm{R}$ & $\mathrm{LC}$ \\
\hline \multicolumn{6}{|c|}{ Capitonidae } \\
\hline 70 & Coppersmith Barbet & Megalaima haemacephala & $\mathrm{UC}$ & $\mathrm{R}$ & $\mathrm{LC}$ \\
\hline \multicolumn{6}{|c|}{ Picidae } \\
\hline 71 & $\begin{array}{l}\text { Yellow-fronted Pied Wood- } \\
\text { pecker }\end{array}$ & Dendrocopos mahrattensis & $\mathrm{UC}$ & $\mathrm{R}$ & $\mathrm{LC}$ \\
\hline \multicolumn{6}{|c|}{ Alaudidae } \\
\hline 72 & Red-winged Bush-Lark & Mirafra erythroptera & $\mathrm{UC}$ & $\mathrm{R}$ & $\mathrm{LC}$ \\
\hline \multicolumn{6}{|c|}{ Hirundinidae } \\
\hline & Streak-throated Swallow & Hirundo fluvicola & RA & $\mathrm{R}$ & $\mathrm{LC}$ \\
\hline \multicolumn{6}{|c|}{ Motacillidae } \\
\hline 74 & White Wagtail & Motacilla alba & $\mathrm{A}$ & WM & $\mathrm{LC}$ \\
\hline 75 & Large Pied Wagtail & Motacilla maderaspatensis & $\mathrm{C}$ & $\mathrm{R}$ & $\mathrm{LC}$ \\
\hline 76 & Citrine Wagtail & Motacilla citreola & $\mathrm{C}$ & WM & $\mathrm{LC}$ \\
\hline 77 & Grey Wagtail & Motacilla cinerea & $\mathrm{C}$ & WM & $\mathrm{LC}$ \\
\hline 78 & Paddyfield Pipit & Anthus rufulus & $\mathrm{UC}$ & $\mathrm{R}$ & LC \\
\hline Pyc & & & & & \\
\hline 79 & Red-vented Bulbul & Pycnonotus cafer & A & $\mathrm{R}$ & $\mathrm{LC}$ \\
\hline 80 & White-eared Bulbul & Pycnonotus leucotis & $\mathrm{UC}$ & WM & $\mathrm{LC}$ \\
\hline Lar & & & & & \\
\hline 81 & Bay-backed Shrike & Lanius vittatus & $\mathrm{UC}$ & $\mathrm{R}$ & $\mathrm{LC}$ \\
\hline Mu & & & & & \\
\hline 82 & Bluethroat & Luscinia svecica & RA & WM & $\mathrm{LC}$ \\
\hline 83 & Oriental Magpie-Robin & Copsychus saularis & $\mathrm{C}$ & $\mathrm{R}$ & $\mathrm{LC}$ \\
\hline 84 & Indian Robin & Saxicoloides fulicata & $\mathrm{UC}$ & $\mathrm{R}$ & $\mathrm{LC}$ \\
\hline 85 & Pied Bushchat & Saxicola caprata & UC & $\mathrm{R}$ & $\mathrm{LC}$ \\
\hline 86 & Indian Chat & Cercomela fusca & A & $\mathrm{R}$ & $\mathrm{LC}$ \\
\hline 87 & Common Babbler & Turdoides caudatus & $\mathrm{UC}$ & $\mathrm{R}$ & $\mathrm{LC}$ \\
\hline
\end{tabular}

Contd.... 
Girish Chopra et al. / J. Appl. \& Nat. Sci. 9 (3): 1475 - 1481 (2017)

\begin{tabular}{|c|c|c|c|c|c|}
\hline 88 & Large Grey Babbler & Turdoides malcolmi & $\mathrm{C}$ & $\mathrm{R}$ & LC \\
\hline 89 & Jungle Babbler & Turdoides striatus & A & $\mathrm{R}$ & LC \\
\hline 90 & Ashy Prinia & Prinia socialis & $\mathrm{C}$ & $\mathrm{R}$ & LC \\
\hline 91 & Plain Prinia & Prinia inornata & $\mathrm{C}$ & $\mathrm{R}$ & LC \\
\hline 92 & Common Tailorbird & Orthotomus sutorius & $\mathrm{C}$ & $\mathrm{R}$ & LC \\
\hline 93 & Common Chiffchaff & Phylloscopus collybita & A & WM & LC \\
\hline \multicolumn{6}{|c|}{ Nectariniidae } \\
\hline \multicolumn{5}{|c|}{ Zosteropidae } & LC \\
\hline Emberizidae & Oriental White-eye & Zosterops palpebrosus & $\mathrm{UC}$ & $\mathrm{R}$ & LC \\
\hline $\begin{array}{l}96 \\
\text { Pass }\end{array}$ & Spotted Munia & Lonchura punctulata & $\mathrm{UC}$ & $\mathrm{R}$ & LC \\
\hline 97 & House Sparrow & Passer domesticus & A & $\mathrm{R}$ & LC \\
\hline 98 & Baya Weaver & Ploceus philippinus & UC & $\mathrm{R}$ & LC \\
\hline \multicolumn{6}{|c|}{ Sturnidae } \\
\hline 99 & Common Myna & Acridotheres tristis & A & $\mathrm{R}$ & LC \\
\hline 100 & Bank Myna & Acridotheres ginginianus & A & $\mathrm{R}$ & LC \\
\hline \multicolumn{6}{|c|}{ Dicruridae } \\
\hline \multicolumn{6}{|c|}{ Corvidae } \\
\hline 102 & Indian Treepie & Dendrocitta vagabunda & $\mathrm{C}$ & $\mathrm{R}$ & LC \\
\hline 103 & House Crow & Corvus splendens & A & $\mathrm{R}$ & LC \\
\hline 104 & Jungle Crow & Corvus macrorhynchos & UC & $\mathrm{R}$ & LC \\
\hline
\end{tabular}

A- Abundant, C- Common, UC- Uncommon, RA- Rare, R- Resident, WM- Winter migrant, SM- Summer migrant, LC- Least concerned, NT- Near threatened, V- Vulnerable

Table 2. Showing Per cent composition and number of bird species in each represented avian order and family in Bhindawas bird sanctuary.

\begin{tabular}{|c|c|c|c|c|}
\hline S.N. & Order & Number (\%) of species & Family & Number (\%) of species \\
\hline 1 & Podicipediformes & $1(0.96 \%)$ & Podicipedidae & $1(0.96 \%)$ \\
\hline \multirow[t]{2}{*}{2} & Pelecaniformes & $4(3.85 \%)$ & Phalacrocoracidae & $3(2.89 \%)$ \\
\hline & & & Anhingidae & $1(0.96 \%)$ \\
\hline \multirow[t]{3}{*}{3} & Ciconiiformes & $11(10.58 \%)$ & Ardeidae & $7(6.73 \%)$ \\
\hline & & & Ciconiidae & $1(0.96 \%)$ \\
\hline & & & Threskiornithidae & $3(2.89 \%)$ \\
\hline 4 & Anseriformes & $13(12.5 \%)$ & Anatidae & $13(12.5 \%)$ \\
\hline 5 & Falconiformes & $4(3.85 \%)$ & Accipitridae & $4(3.85 \%)$ \\
\hline 6 & Galliformes & $2(1.92 \%)$ & Phasianidae & $2(1.92 \%)$ \\
\hline \multirow[t]{2}{*}{7} & Gruiiformes & $5(4.81 \%)$ & Gruidae & $2(1.92 \%)$ \\
\hline & & & Rallidae & $4(3.85 \%)$ \\
\hline \multirow[t]{4}{*}{8} & Charadriiformes & $12(11.54 \%)$ & Jacanidae & $2(1.92 \%)$ \\
\hline & & & Charadriidae & $3(2.89 \%)$ \\
\hline & & & Scolopacidae & $5(4.81 \%)$ \\
\hline & & & Recurvirostridae & $2(1.92 \%)$ \\
\hline 9 & Columbiformes & $5(4.81 \%)$ & Columbidae & $5(4.81 \%)$ \\
\hline 10 & Psittaciformes & $2(1.92 \%)$ & Psittacidae & $2(1.92 \%)$ \\
\hline 11 & Cuculiformes & $2(1.92 \%)$ & Cuculidae & $2(1.92 \%)$ \\
\hline 12 & Strigiformes & $1(0.96 \%)$ & Strigidae & $1(0.96 \%)$ \\
\hline \multirow[t]{5}{*}{13} & Coraciiformes & $7(6.73 \%)$ & Alcedinidae & $2(1.92 \%)$ \\
\hline & & & Meropidae & $2(1.92 \%)$ \\
\hline & & & Coraciidae & $1(0.96 \%)$ \\
\hline & & & Upupidae & $1(0.96 \%)$ \\
\hline & & & Bucerotidae & $1(0.96 \%)$ \\
\hline \multirow[t]{2}{*}{14} & Piciformes & $2(1.92 \%)$ & Capitonidae & $1(0.96 \%)$ \\
\hline & & & Picidae & $1(0.96 \%)$ \\
\hline \multirow[t]{13}{*}{15} & Passeriformes & $33(31.73 \%)$ & Alaudidae & $1(0.96 \%)$ \\
\hline & & & Hirundinidae & $1(0.96 \%)$ \\
\hline & & & Motacillidae & $5(4.81 \%)$ \\
\hline & & & Pycnonotidae & $2(1.92 \%)$ \\
\hline & & & Laniidae & $1(0.96 \%)$ \\
\hline & & & Muscicapidae & $12(11.54 \%)$ \\
\hline & & & Nectariniidae & $1(0.96 \%)$ \\
\hline & & & Zosteropidae & $1(0.96 \%)$ \\
\hline & & & Emberizidae & $1(0.96 \%)$ \\
\hline & & & Passeridae & $2(1.92 \%)$ \\
\hline & & & Sturnidae & $2(1.92 \%)$ \\
\hline & & & Dicruridae & $1(0.96 \%)$ \\
\hline & & & Corvidae & $3(2.88 \%)$ \\
\hline
\end{tabular}


also reported such as Darter, Black-necked Stork, Oriental White Ibis, Alexandrine Parakeet, Common Pochard and Sarus Crane. It is also recommended that the research related to diversity, abundance, residential status and migration patterns of birds should be regularly carried out, so that the reduction in diversity, population size and their ecological reasons can be determined.

\section{REFERENCES}

Abbas, S., Tabassum, R., Khan, M.Z., Khan, B., Hussain, S., Khan, G. and Awan, S. (2014). Avian diversity in Central Karakoram National Park, Gilgit-Balistan. International Journal of Agriculture and Biology. 16(2):377382

Ali, S. and Ripley, S.D. (1987) Handbook of birds of India and Pakistan, Compact Edition, Oxford University Press, Mumbai

Aravind, N.A., Rao, D. and Madhusudan, P.S. (2001). Additions to the birds of Biligiri Rangaswamy temple wildlife sanctuary, Western Ghats, India. Zoo's Print Journal. 16 (7) :541-547

Barsal, H.S. and Inskipp, C. (2009). The birds of Sukla Phanta wildlife reserve, Nepal. Our Nature. 7:56-81

Barua, M. and Sharma, P. (1999). Birds of Kaziranga National Park, India. Foktail. 15:47-60

Beresford, P., Barker, F.K., Ryan, P.G. and Crowe, T.M. (2005). African endemics span the tree of songbirds (Passeriformes): molecular systematic of several evolutionary "enigmas". Proceedings of the Royal Society B: Biological Sciences. $272: 849-858$

Bibi, F. and Ali, Z. (2013). Measurement of diversity indices of avian communities at Taunsa barrage wildlife sanctuary, Pakistan. The Journal of Animal \& Plant Sciences. 23 (2) : 469-474

BirdLife International (2016) Country profile: India. Available from: http://www.birdlife.org/datazone/country/ india. Checked: 2016-05-22

Chilke, A.M. (2012). Avian diversity in and around Bamanwada lake of Rajpura, district Chandrapur (Maharashtra). Annals of Biological Research. 3 (4) :2014-2018

Chopra, G. and Sharma, S.K. (2014). Avian diversity of lower Shivalik foothills, India. International Journal of Research Studies in Biosciences. 2 (7) :1-12

Deka, C. and Nath, B. (2013). A study on avifaunal diversity and their conservation status of Chandubi Tectonic lake, Assam, India. International Journal of Pure \& Applied Bioscience. 1 (6) :67-71

Dhadse, S., Kotangale, J.P., Chaudhari, P.R. and Wate, S.R. (2009). Diversity of birds at Panipat refinery, Haryana (India). Environmental Monitoring and Assessment. $158: 1-8$
Gaston, A.J. (1975). Methods for estimating bird population. The Journal of the Bombay Natural History Society. 69 (3) : 591-615

Ghorade, I.B., Thakur, V.R. and Patil, S.S. (2014). Diversity of avian fauna from Jaikwadi reservoir at Paithan. European Academic Research. 2 (2) : 1967-1978

Grimmett, R., Inskipp, C. and Inskipp, T. (1999). Pocket guide to the birds of the Indian subcontinent. Oxford University Press, Delhi, India

Harisha, M.N. and Hosetti, B.B. (2009). Diversity and distribution of avifauna of Lakkavalli range forest, Bhadra wildlife sanctuary, Western Ghat, India. Ecoprint. $16: 21-27$

Indika, J. and Mahaulpatha, D.W.A. (2015). Patterns of seasonal abundance and diversity in the waterbird community of Anavilundawa sanctuary. Wildlanka. 3(1):31-37

Kumar, J. I.N. (2007). Pattern of seasonal abundance and diversity in the waterbird community of Nal lake bird sanctuary, Gujrat, India. Bird Populations. $8: 1-20$

Lameed, G.A. (2012). Species diversity and richness of wild birds in Dagona-waterfowl sanctuary, Nigeria. African Journal of Food, Agriculture, Nutrition and Development. 12 (5) :6461-6478

Mahabal, A. (2000). Birds of Talra wildlife sanctuary in lower Western Himalaya, H. P., with notes on their status and attitudinal movements. Zoo's Print Journal. 15 (10) :334-338

Maity, S., Balachandran, S. and Chaudhury, S. (2010). Interdependency of macrophytes and avian diversity in the wetlands of Ballavpur wildlife sanctuary, Santiniketan. Science and Culture. 76 (5-6): :180-184

Manakandan, R. and Pittie, A. (2001). Standardized common and scientific names of the birds of the Indian Subcontinent. Buceros. 6 (1) : i-ix, 1-37

Pawar, P.R. (2011). Species diversity of birds in mangroves of urban (Raigad), Navi Mumbai, Maharashtra, West coast of India. Journal of Experimental Sciences. 2 (10) :73-77

Saikia, P.K. and Saikia, M.K. (2011). Present distribution, status, and ecology of White-winged Duck and Hornbills in Nameri National Park, considering the tropical forest distrinution of Assam. Zoo's Print. 24 :1-11

Sale, J.B. and Berkmuller, K. (1988). Manual of wildlife techniques for India. Field document No.11. FAO, United Nations, Dehradun, India, pp 243

Srinivasulu, C. and Nagulu, V. (2001). Mammalian and Avian diversity of the Nallamala Hills, Andhra Pradesh. Zoo's Print Journal. 17 (1) : 675-684.

Urfi, A.J. (2003). The birds of Okhla barrage bird sanctuary Delhi, India. Forktail. $19: 39-50$

Wijesundara, C. and Wijesundara, M. (2014). Bird diversity of Dekinda forest reserve, Balana, Sri Lanka: Implications for conservation. Ceylon Journal of Science, 43 (1) : 137-146 